\title{
Análise do Desempenho Computacional de Modelos Numéricos de Previsão do Tempo e de Ondas na Arquitetura EC2 Amazon Cloud
}

\author{
Marcos Vinícius Bueno de Morais; Alex Sandro Aguiar Pessoa; Sandra Isay Saad; \\ Luiz Felipe Xavier; Camila Gomes Martins Ramos; Bianca Lobo; Gilca Palma
}

Grupo de Modelagem, Pesquisa e Desenvolvimento, CLIMATEMPO

04011-061, São Pau lo, SP

\begin{abstract}
E-mail:mvbueno@climatempo.com.br, alex.pessoa@climatempo.com.br, sandra@climate mpo.com.br, camila@climatempo.com.br,bianca@climatempo.com.br, gilca@climatempo.com.br, luiz.xavier@climatempo.com.br
\end{abstract}

\section{RESUMO}

Com os constantes avanços dos modelos numéricos de previsão de tempo e ondas, na execução de grades cada vez mais refinadas, sempre houve a necessidade de utilização e rápida atualização dos equipamentos e servidores que executam estes modelos, de tal forma a agilizar a previsão e efetuar cálculos com maior precisão e acurácia. A computação em nuvens, com a habilidade de virtualização de CPU's e a facilidade de manuse io nos servidores, permite que os mode los mais atuais sejam executados de forma cada vez mais rápida e precisa. Por isso, diversas empresas e comunidades científicas têm substituído os tradicionais servidores físicos pelo uso de computação nas nuvens, para o desenvolvimento de projetos e até no uso de computação de alto desempenho operacional.

Este trabalho visou avaliar o desempenho dos modelos numéricos WRF (Weather Research and Forecast, [3]), na sua versão 3.5, e WW3 (WaveWatch - 3rd Generation, [5]), na versão 3.14, que são rodados operacionalmente na Climatempo, em instâncias de grande uso de processamento da Amazon Web Services (AWS). Estes modelos têm apresentado bons resultados quantitativos na previsão de tempo e onda [4]. A unidade selecionada é otimizada para computação de alto desempenho, com codinome $c c 2.8 x l a r g e$, que tem proporcionalmente mais recursos de CPU do que memória (RAM) e são adequadas para aplicativos com processamento intensivo. Esta instância conta com 60,5 Gb de memória RAM, 88 unidades de processamento EC2, 3370 GB de armazenamento de instâncias locais, plataforma de 64 bits e conexão Ethernet de 10 Gigabits. Os processadores são Intel ${ }^{\circledR}$ Xeon ${ }^{\circledR}$ CPU E5-2670 $0 @ 2.60$ Ghz, tota lizando 32 núcleos (16 físicos e 16 virtuais).

O modelo numérico de previsão do tempo foi configurado para um passo de tempo de $30 \mathrm{~s}$, com 4 grades no total, sendo a grade-mãe, abrangendo a costa brasileira, com $39 \mathrm{~km}$ de espaçamento horizontal, e as outras 3 grades separadas para as regiões nordeste, sudeste e sul da costa, com $13 \mathrm{~km}$. Já o modelo oceânico foi ajustado com 2 grades, sendo a primeira com $1.5^{\circ}$ de espaçamento horizontal e abrange o globo, e a segunda pega toda a costa do atlântica da América do Sul. Ambas as rodadas foram feitas para 24 horas de previsão.

Para avaliação do desempenho de execução em paralelo foram escolhidos, juntamente com o tempo, os índices SpeedUp e Eficiência [2], comumente utilizados para avaliação do desempenho de modelos numéricos de previsão em clusters com vários núcleos [1].

Os resultados demonstraram que o WRF é otimizado utilizando todas as unidades de processamento físico (16 cores). A partir disto, o modelo acaba diminuindo a eficiência não melhorando o tempo ou o índice de SpeedUp. Já para o modelo de ondas, a condição ótima dada com 12 núcleos, com um mínimo de eficiência para 28 núcleos. Na otimização, o tempo de execução da rodada do WRF e do WW3 foi respectivamente de 3.3 min e 6.6 min.

Palavras-chave: Cloud Computing, Paralelismo, Computação de alto desempenho, Previsão Numérica de Tempo, WRF, WW3, Amazon EC2, AW 


\section{Referências}

[1] E. S. Almeida, Evaluation of HPC architectures for BRAMS numerical weather model em "The 2011 World Congress in Computer Science, Computer Engineering and Applied Computing” (M. Bauer e A. L. Fazenda), pp. 1-7, Las Vegas, Nevada, 2011.

[2] D. L. Eager, J. Zahorjan, E.D. Lazowska, Speedup versus efficiency in parallel systems, Computers, IEEE Transactions on , vol. 38, n.3, pp. 408-423, (1989).

[3] J. Michalakes, J. Dudhia, D. Gill, J. Klemp, W. Skamarock, Design of a next-generation regional weather research and forecast model, Towards Teracomputing, World Scientific, River Edge, New Jersey, pp. 117-124, (1998).

[4] S. I. Saad, Avaliação dos Modelos de Previsão de Tempo utilizados na CLIMATEMPO baseado no dia de antecedência da Previsão em "XVI Congresso Brasileiro de Meteorologia" (R. Palmeira e G. Palma), pp. 1-6, Belém, Pará, 2010.

[5] H. L. Tolman, A Third-Generation model for wind waves on slowly varying, unsteady inhomogeneous depths and currents, Journal of Physical Oceanography, vol. 21, pp. 782787, (1990). 